\title{
ORIGINAL ARTICLE \\ Sociodemographic factors associated with sexual dysfunction in Mexican women with spinal cord injury
}

\author{
M Moreno-Lozano ${ }^{1}$, S Durán-Ortíz ${ }^{2}$, R Pérez-Zavala² and J Quinzaños-Fresnedo
}

Objectives: No studies have reported sexual dysfunction in Mexican women with a spinal cord injury (SCI). The objective of the present study was to determine the association between sociodemographic factors and sexual dysfunction characteristics in Mexican females with SCl.

Methods: An observational, cross-sectional, descriptive study was conducted in different rehabilitation centers in Mexico City from July 2013 to November 2014. Adult females with a SCI without any gynecologic structural abnormalities, which by itself produced sexual dysfunction, were included. The Female Sexual Function Index (FSFI) was answered personally by all participants. Other variables such as AIS (American spinal injury association Impairment Scale), neurologic level, time since injury, age, relationship status, socioeconomic status, spasticity, use of antispasticity drugs, education level, antidepressant medication, Spinal Cord Independence Measure III score, offspring, work activities and neuropathic pain were considered.

Results: There is a high percentage of sexual dysfunction among Mexican woman with $\mathrm{SCl}(81.9 \%)$. Age range went from 18- to 78year old (42.8 \pm 15.87-year old). Time since injury went from 2 to 708 months $(65.16 \pm 117.65$ months). The study showed a negative correlation between age and the FSFI questionnaire (correlation coefficient $(C C)=-0.384, P<0.001$ ). There was no significant difference between the means (analysis of variance) of the different groups for neurologic level, socioeconomic status, spasticity and education level.

Conclusion: Results showed that the younger the person is, the better sexual function they have, and offspring decreased sexual function and work activities increased it. Some variables showed small comparative groups (use of antidepressives/antispastics and comorbilities), which may be the reason we could not see significative differences in the means.

Spinal Cord (2016) 54, 746-749; doi:10.1038/sc.2016.19; published online 23 February 2016

\section{INTRODUCTION}

Spinal cord injury (SCI) causes several deficiencies that limit the lives of individuals and their participation in society, and hence its global impact is important. Multiple changes arise from the neurologic injury suffered by these patients. These changes limit their physical activity, alter their psychological state, impact their economy, and restrict social integration and previous life projects at different levels. ${ }^{1}$ Patients eventually understand these changes and understand that walking is not always the most important function to recover in order to improve their quality of life. They understand that a good bladder and bowel function, adequate autonomic dysreflexia identification and managing skills, upper limb motor activity improvement, as well as a return to sexual activity regardless of the level of injury have a major role in their future quality of life. ${ }^{1}$

Sexual function is impaired in women with SCI due to motor, sensory and autonomic involvement; this has a significant impact on different areas of their lives. ${ }^{2,3}$

According to the International Consensus Development Conference on Female Sexual Dysfunction, sexual dysfunction implies personal distress; hence, a woman with a SCI who has alterations in her sexual response but has no signs of distress would not be considered to have a sexual dysfunction. ${ }^{4,5}$

We can establish that a neurologic injury can determine different levels of sexual dysfunction among women with SCI (this is known as primary effect); this negative effect is amplified by related problems such as neurogenic bowel and bladder, spasticity, pain and psychological aspects such as low self-esteem/self-image or personal relationship issues, which can affect sexual activity, sexual satisfaction and sexual response (secondary effect). This leads in turn to failures in social interpersonal relationships (tertiary effect). ${ }^{6}$

In a review conducted in patients with SCI in which their sexual function was assessed, it was found that marriage and strong relationships had a better prognosis for obtaining a better sexual adjustment. There is some controversy as to whether the age of onset of a SCI has a significant impact on sexuality. FerreiroVelasco et al. ${ }^{7}$ found in their study that women under 18 years of age had a higher risk of not establishing future sexual relationships $(P=0.04)$.

As for the etiology of SCI, several studies report that female patients with traumatic spinal cord injuries achieve greater improvement compared with those with non-traumatic injuries. McKinley reported

${ }^{1}$ Instituto Nacional de Rehabilitación, Médico Residente de Cuarto Año, Medicina de Rehabilitación, México DF, Mexico and ${ }^{2}$ Instituto Nacional de Rehabilitación, División de Rehabilitación Neurológica, México DF, Mexico

Correspondence: Dr J Quinzaños-Fresnedo, Instituto Nacional de Rehabilitación, División de Rehabilitación Neurológica, Avenida México-Xochimilco No. 289, Col. Arenal de Guadalupe, Dlegación Tlalpan, México DF CP 14389, Mexico.

E-mail: jimenaqf@hotmail.com

Received 26 November 2015; revised 12 January 2016; accepted 14 January 2016; published online 23 February 2016 
a higher incidence of autonomic dysreflexia ( $0 \%$ vs $24.1 \%)$, spasticity $(21.1 \%$ vs $44.3 \%)$, depression $(23.7 \%$ vs $26.6 \%)$, urinary tract infections $(52.6 \%$ vs $67.1 \%)$ and pain $(55.3 \%$ vs $62 \%)$ in patients with non-traumatic etiology, and all of these are known conditions that interfere with sexual activity and function. ${ }^{6}$

Some studies have shown that 40 to $80 \%$ of women remain sexually active after a SCI. Ferreiro-Velasco et al. reported a significant decrease in sexual activity frequency after a SCI (from 9.9 to 4.2 times per month). The literature also reports that as time from initial injury goes by, women are able to achieve a better sexual satisfaction $(P<0.02){ }^{6}$

There are also associated comorbidities reported in women with SCI, such as increased risk for diabetes mellitus (three times higher than the general population) and secondary cardiovascular disease that can develop from a prior metabolic syndrome. ${ }^{6}$

It is important to keep monitoring these patients by performing regular ASIA scale exams during the first 3 months after presenting the injury, mainly because it has been observed that most of the improvement occurs at this time, and it can correlate with a better future sexual adjustment. ${ }^{6}$

A consistent finding in several studies is that bladder or anal incontinence is a major limiting factor in women with SCI while having any kind of sexual experience. The fear of incontinence that these patients experience can also affect them psychologically, and all of these becomes a feedback between the previously discussed factors; some precautions to take such as emptying the bladder and bowel before engaging in sexual activity should be considered. ${ }^{8,9}$

The rehabilitation of sexual function in women with SCI is focused on facilitating their sexual expression in order for this one to become acceptable and satisfying for them, by understanding that sexual satisfaction is a dynamic process, one that is subject to continuous development through time, hence it is necessary to conduct a continuous approach. ${ }^{10}$

\section{MATERIALS AND METHODS}

An observational, cross-sectional and descriptive study was conducted at different rehabilitation centers in Mexico City from July 2013 to March 2015. The study used the following inclusion criteria: being a Mexican woman with a SCI diagnosis, older than 18 years of age and accepted to form part of the present study by signing a written consent. Exclusion criteria were as follows: any gynecologic structural abnormalities that produced sexual dysfunction by its own, history of mental deficiency and being a non-Spanish speaker. Sample size was calculated with the Epidat 4 software program (Galicia, Spain). On the basis of a previous study, ${ }^{1}$ where a correlation of female sexual function with chronic pain was 0.28 , and with spasticity was 0.22 , we performed a sample-size calculation. A total of $77-126(0.28$ and 0.22 , respectively) patients are required for this correlation, a study power of $80 \%$ and a $95 \%$ confidence interval.

We applied the Female Sexual Function Index (FSFI) questionnaire, which is a multidimensional instrument validated in Spanish. It contains 19 questions (divided into six areas) assessing female sexual function in the previous 4 weeks. It has a minimum score of 2 and a maximum of 36 . Scores above 26.55 mean that the patient does not have a sexual dysfunction. ${ }^{11,12}$

Other variables collected were AIS, neurologic level, time since injury, age, relationship status, socioeconomic status, spasticity and use of antispasticity drugs, education level, use of antidepressant medication, Spinal Cord Independence Measure version III score, offspring, work activities, neuropathic pain, comorbidities and etiology.

For the statistical analysis, we used descriptive measures for sociodemographic variables; we applied a Pearson correlation, analysis of variance and a $t$-test to identify the association between sexual dysfunction and the other variables, with a $95 \%$ confidence interval. $P$ values of $<0.05$ were considered as statistically significant.

\section{RESULTS}

We interviewed 128 women with SCI, but 45 women did not agree to form part of the study because of lack of interest in answering the questionnaire or for unspecified reasons, leaving a total sample of 83 patients who met the inclusion criteria.

The age of our patients ranged from 18 to 78 years of age $(42.8 \pm 15.87$-year old). The time since SCI injury ranged from 2 to 708 months (59 years), with a mean of $65.16 \pm 117.65$ months.

In relation with the AIS, 40 women corresponded to AIS A, 10 women to AIS B, 12 women to AIS C, 19 women to AIS D and 2 women to AIS E.

Regarding the neurological level of injury, we encountered that 16 women had a cervical level, 26 had a high thoracic level, 34 had a low thoracic level and 7 had a lumbar level.

The average FSFI questionnaire score found in our study was $11.53 \pm 11.26$. Our results show a high percentage of sexual dysfunction among Mexican woman with SCI (81.9\%).

Regarding socioeconomic status, 20 women had no economic resources, 2 had very low resources, 25 with low resources, 31 as medium-low resources, 1 medium and 1 medium-high resource availability.

Regarding spasticity (measured by the modified Ashworth scale), 49 patients had a grade 0 muscle tone, 19 patients had a grade 1 muscle tone, 9 patients had a grade 2 muscle tone and 6 patients had a grade 3 muscle tone.

We found that the level of education in our sample corresponds mostly to women with a bachelor's degree $(n=26)$, followed by high school level $(n=21)$, junior high $(n=18)$, elementary school $(n=17)$ and none $(n=1)$.

For dichotomous variables, we found that 38 women had a partner and 45 women did not have a partner. Regarding the use of medication, we found that 67 women used antidepressants and 68 women used antispasticity drugs.

Forty-nine women had children and 33 did not; however, none of the women had children after their SCI. At the moment of the study, only 21 women had work, the rest of them $(N=62)$ did not work. Forty-one women had neuropathic pain. Only 28 women had comorbidities (hypertension, diabetes mellitus and thyroid disorders).

According to the SCI etiology, most were traumatic $(n=47)$ compared with non-traumatic etiology $(n=36$; see Table 1$)$.

We used Pearson's Correlation for FSFI and age, as well as for time since injury and Spinal Cord Independence Measure III score; the study showed a negative correlation between age and FSFI questionnaire score $(\mathrm{CC}=-0.384, P<0.001)$ without observing associations between the FSFI score and the time since injury $(\mathrm{CC}=0.087$, $P=0.436)$, or with Spinal Cord Independence Measure III score (CC $=0.160, P=0.149$; see Table 2).

Analysis of variance was used for parametric variables without observing statistically significative differences between the mean FSFI score and the neurological level (average 95.006, $P=0.568$ ), between

\section{Table 1 Frequencies}

\begin{tabular}{lcc}
\hline Variable & $\mathrm{n}$ & $\%$ \\
\hline Sexual dysfunction & 68 & 81.9 \\
AIS (A,B,C,D and E) & $40 / 10 / 12-$ & $48.2 / 12 / 14.5 / 22.9 / 2.4$ \\
& $/ 19 / 2$ & \\
Neurologic level (cervical,high thoracic, & $16 / 26 / 34 / 7$ & $19.3 / 31.3 / 41 / 8.4$ \\
low thoracic and lumbar) & & \\
Etiology (traumatic/non-traumatic) & $47 / 36$ & $56.6 / 43.4$ \\
\hline
\end{tabular}


Table 2 Correlations

\begin{tabular}{lcr}
\hline Female Sexual Function Index & CC & P-value \\
\hline Age & $-\mathbf{0 . 3 8 4}$ & $<0.001$ \\
Evolution time since injury & 0.087 & 0.436 \\
SCIM III & 0.160 & 0.149 \\
\hline
\end{tabular}

Abbreviation: Spinal Cord Independence Measure.

Statistically significant values are indicated in bold.

FSFI score and socioeconomic status (average 148.929, $P=0.322$ ), FSFI score and spasticity (average 106.174, $P=0.529$ ), and FSFI score and education level (average 48.462, $P=0.772$ ).

We used a $t$-test for dichotomous parametric variables such as antidepressants, antispasticity drugs, family, children and work. The average IFSF score for those without children was $16.12 \pm 12.17$ and those who do have children was $8.50 \pm 9.58$, with a significant difference between groups $(P=0.002)$. The average FSFI score in the non-worker group was $9.37 \pm 10.51$ and in the working group was $17.9 \pm 11$, with a statistically significant difference between groups $(P=0.002)$. For the rest of the variables, we did not find a statistically significant difference between groups, although there is a tendency for those not using antidepressants, those who have a partner and those who have no pain to achieve higher FSFI scores as summarized below. (see Table 3).

\section{DISCUSSION}

SCI generates a negative impact on various aspects of life; in this study, we focused on the sexual aspect. Other studies have reported a number of factors that take an impact on sexual function in patients with SCI, such as the neurologic level, ASIA scale, age, time since injury, relationship status, neuropathic pain, use of antidepressants or antispasticity drugs and etiology.

This study shows a high percentage of sexual dysfunction $(81.9 \%)$ in Mexican women with SCI in contrast to what has been reported in other countries (40-50\%). However, we must emphasize that there is a high prevalence of sexual dysfunction in Mexican women without any pathology $(52.2 \%)$; hence, it is expected for women with a SCI to present higher prevalence of sexual dysfunction.

We also took into consideration that the FSFI questionnaire applies only to the previous 4 weeks from the time it is answered; this fact plus the diminished frequency of sexual activity can explain the 'high percentage' of sexual dysfunction in our sample.

In relation to the ASIA scale, most injuries in our study are scale A; hence, sexual response is altered, which helps explain the higher percentage of sexual dysfunction that we report.

Regarding the neurological level, those with a cervical level may be at an increased risk of sexual dysfunction due to the presence of complications and diminished participation in general and sexual activities. On the other hand, Matzaroglou et al. ${ }^{13}$ reported no difference with the level of injury, which is consistent with what we found in our population.

In this study, the factors significantly associated with sexual function are patient age (the younger the patient is, the lower the risk of presenting sexual dysfunction), as is reported by Bering, having offspring puts these specific patients at a greater risk for having sexual dysfunction, whereas being employed correlates with a better sexual function. Apparently, having a work activity gives an adequate support network to the patient and gives a good return to sexual activity
Table $3 t$-test

\begin{tabular}{lccccc}
\hline Variable & & & & & \\
Antidepressants & No & 67 & $12.11 \pm 11.49$ & 0.964 & 0.338 \\
& Yes & 16 & $9.09 \pm 10.17$ & & \\
Antispasticity drugs & No & 68 & $11.48 \pm 11.17$ & -0.085 & 0.932 \\
& Yes & 15 & $11.76 \pm 12.02$ & & \\
Partner & No & 38 & $10.12 \pm 10.89$ & -1.051 & 0.296 \\
& Yes & 45 & $12.72 \pm 11.54$ & & \\
Offspring & No & 33 & $16.12 \pm 12.17$ & 3.181 & 0.002 \\
Work activities & Yes & 50 & $8.50 \pm 9.58$ & & \\
Pain & No & 62 & $9.37 \pm 10.51$ & -3.157 & 0.002 \\
& Yes & 21 & $17.90 \pm 11.20$ & & \\
Comorbidities & No & 42 & $13.24 \pm 12.07$ & 1.408 & 0.163 \\
& Yes & 41 & $9.78 \pm 10.21$ & & \\
Etiology & No & 55 & $11.31 \pm 11.33$ & -0.243 & 0.809 \\
& Yes & 28 & $11.95 \pm 11.31$ & & \\
\hline
\end{tabular}

Statistically significant values are indicated in bold.

prognosis; we find this very interesting and it makes us emphasize the importance of getting them back to work-related activities (given that in our study only 21 women engaged in some type of work activity).

As for the time since injury, in our study no association was found between this and the presence of sexual dysfunction contrary to what was reported in the Biering study, ${ }^{2}$ where it was found that a longer history of SCI increased sexual activity or satisfaction; this is because of greater freedom to express their sexuality and higher self-knowledge. It is possible that we did not find no correlation with this variable because of such a wide range of time since injury in our sample of patients (months to years), and the previously mentioned study was conducted in people with a history of 10 years or more of living with a SCI.

We also found no association with the use of different drugs; this is probably due to the differences between our groups; hence, it would be advisable to try to make a more homogeneous sample.

It has been reported that the presence of comorbidities such as diabetes mellitus or metabolic syndrome affects female sexual function; in this study, a direct correlation between the reported comorbidities (hypertension, heart disease and asthma) and sexual function was observed. The reason for this may be that we did not assess whether women already had one of these comorbidities before sustaining a SCI; hence, there is a chance that they could have already presented a sexual dysfunction before their injury, and in addition there is also a significant difference in the number of participants in both groups (most women did not present any comorbidities).

Regarding etiology, it has been observed that in other studies traumatic SCI patients have a better sexual function prognosis, and this study found no such association.

Despite not having found a significant difference between the FSFI score and socioeconomic status, educational level, antidepressant medication intake, the presence of a partner and the absence of pain, we feel that there is a tendency for these variables to be associated with a better sexual function. This trend would probably have statistical significance if a larger number of the women studied had a good sexual function. 


\section{Limitations of the study}

The main limitation of the study is that there are not enough women with adequate sexual function in Mexico, which limits the association with the variables studied in this work.

Although women were sought in non-hospital populations (Grupo Altía IAP (Institución de Asistencia Privada); independent living), most of the people studied were patients of the National Institute of Rehabilitation 'Luis Guillermo Ibarra Ibarra', which translates to a selection bias.

Finally, some variables show very homogeneous distributions and other very small comparison groups (antidepressants/antispasticity drug use and comorbidities), not enough to observe statistically significant differences in mean scores; hence, it would be necessary to increase the sample size within groups.

\section{Strengths of the study}

We have a wide sample of variables in our present study in comparison with other studies. During this study, we saw some factors that strongly influence the female sexual function in women with SCI and some trends in other variables, which encourage us to increase the number of women without sexual dysfunction in the sample in order for us to establish significant correlations. Having knowledge of these variables, we can make an intervention in the hopes of having a positive impact on the female sexual function.

\section{CONCLUSIONS}

There is a high percentage of sexual dysfunction in Mexican women with SCI with different factors that influence this in addition to the changes that occur secondary to the SCI itself. In this study, the associated factors were age, offspring and work activity.

It is known that, according to the priorities that spinal cordinjured patients have, sexual aspect ranks first or second, followed by proper bladder and bowel management, as well as motor function; hence, this aspect should be assessed regularly in order for us to give an integral therapeutic approach and improve the quality of life of our patients.

\section{DATA ARCHIVING}

There were no data to deposit.

\section{CONFLICT OF INTEREST}

The authors declare no conflict of interest.

1 Anderson K, Borisoff J, Johnson R, Stiens S, Elliott S. The impact of spinal cord injury on sexual function: concerns of the general population. Spinal Cord 2007; 45: 328-337.

2 Biering I, Bolling R, Biering F. Sexual function in a traumatic spinal cord injured population 10-45 years after injury. J Rehabil Med 2012; 44 926-931.

3 Kreuter M, Siösten A, Bierong-Sorensen F. Sexuality and sexual life in women with spinal cord injury: a controlled study. J Rehabil Med 2008; 40: 61-69.

4 Sipski M, Arenas A. Female sexual function after spinal cord injury. Prog Brain Res 2006; 152: 441-447.

5 Alexander M, Biering F, Elliot S, Krueter M, Sonksen J. International spinal cord injury female sexual and reproductive function basic data set. Spinal Cord 2011; 49: 787-790.

6 Lombardi G, Del Popolo G, Macchiarella A, Mencarini M, Celso M. Sexual rehabilitation in women with spiNal cord injury: a critical review of literature. Spinal Cord 2010; 48: 842-849.

7 Ferreiro-Velasco ME, Barca-BuyoA, Salvador de la Barrera S, Montoto-Marqués A, Miguéns Vázquez $X$, Rodríguez-Sotillo $A$. Sexual issues in a sample of women with spinal cord injury. Spinal Cord 2005; 43: 51-55.

8 Abramson C, McBride K, Konnyu K, Elliott S. Sexual health outcome measures for individuals with spinal cord injury: a systematic review. Spinal Cord 2008; 46: 320-324.

9 Anderson K, Borisof J, Stiens S, Elliott S. Spinal Cord injury influences psychogenic as well as physical components of female sexual ability. Spinal Cord 2007; 45: 349-359.

10 Forsythe E, Horsewell J. Sexual rehabilitation of women with a spinal cord injury. Spinal Cord 2006; 44: 234-241.

11 Blümel J, Binfa L, Cataldo P, Carrasco A, Izaguirre H, Sarrá S. Índice de Función Sexual Femenina: Un test para evaluar la sexualidad de la mujer. Rev Chil Obstet Ginecol 2004; 69: 118-125.

12 Sipski M, Brackett N, Bodner D, Elliot S, Jackson A, Sonksen J. Measurement of sexual functioning after spinal cord inury: preferred instruments. J Spinal Cord Med 2009; 32 226-236.

13 Matzaroglou Ch, Assimakopoulos K, Panagiotopoulos E, Kasimatis G, Dimakopoulos P, Lambris $E$. Sexual function in females with severe cervical spinal cord injuries: a controlled study with the Female Sexual Function Index. Int J Rehabil Res 2005; 28: 375-377. 